\title{
Points to consider: is there evidence to support BRCA1/2 and other inherited breast cancer genetic testing for all breast cancer patients? A statement of the American College of Medical Genetics and Genomics (ACMG)
}

\author{
Tuya Pal, MD ${ }^{1}$, Doreen Agnese, $\mathrm{MD}^{2}$, Mary Daly, MD, PhD ${ }^{3}$, Albert La Spada, MD, PhD ${ }^{4}$ \\ Jennifer Litton, $\mathrm{MD}^{5}$, Myra Wick, MD, $\mathrm{PhD}^{6}$, Susan Klugman, MD7, Edward D. Esplin, MD, PhD ${ }^{8}$ and \\ Gail P. Jarvik, MD, PhD ${ }^{9}$; on behalf of the Professional Practice and Guidelines Committee
}

Disclaimer: This points to consider document is designed primarily as an educational resource for medical geneticists and other clinicians to help them provide quality medical services. Adherence to this points to consider document is completely voluntary and does not necessarily assure a successful medical outcome. This points to consider document should not be considered inclusive of all proper procedures and tests or exclusive of other procedures and tests that are reasonably directed to obtaining the same results. In determining the propriety of any specific procedure or test, the clinician should apply his or her own professional judgment to the specific clinical circumstances presented by the individual patient or specimen.

Clinicians are encouraged to document the reasons for the use of a particular procedure or test, whether or not it is in conformance with this points to consider document Clinicians also are advised to take notice of the date this points to consider document was adopted, and to consider other medical and scientific information that becomes available after that date. It also would be prudent to consider whether intellectual property interests may restrict the performance of certain tests and other procedures.

Keywords: breast cancer; genetic testing; multigene panels; $B R C A 1 ; B R C A 2$
Genetics in Medicine (2020) 22:681-685; https://doi.org/10.1038/s41436019-0712-x

\section{BACKGROUND}

\section{Breast cancer and genetic testing}

Of all cancers that develop in women in the United States, breast cancer has the highest incidence, regardless of race or ethnicity, with an estimated 271,270 new cases and 42,260 deaths during 2019. ${ }^{1}$ Approximately $5-10 \%$ of breast cancers are estimated to result from hereditary causes, the majority of which are attributed to pathogenic or likely pathogenic (P/LP) variants in the BRCA1 and BRCA2 (BRCA1/2) genes, although other variants in genes such as PALB2, TP53, PTEN, CDH1, CHEK2, and ATM contribute. ${ }^{2}$ Identification of inherited cancer risk empowers individuals and their families to prevent cancers or detect them early. Furthermore, incorporating genetic testing results into patients' care plans has the potential to guide treatment and improve outcomes. ${ }^{3,4}$
Despite the longstanding availability of testing for inherited cancer, including the BRCA1/2 genes for which testing has been clinically available for over two decades, only a small proportion of the at-risk population has been tested. It has been estimated that less than $10 \%$ of all adults with BRCA1/2 $\mathrm{P} / \mathrm{LP}$ variants in the United States have been identified. ${ }^{5}$ Among cancer patients, recent data suggest that less than 20\% of patients with breast or ovarian cancer who meet national criteria for inherited cancer testing actually undergo testing, ${ }^{6}$ often due to lack of identification of high-risk patients and provider recommendations. ${ }^{7-11}$ Moreover, testing rates are disproportionately lower among racial/ethnic minority populations. ${ }^{9,10,12}$ Consequently, there remains a tremendous unmet need to improve identification and testing among high-risk populations and reduce existing barriers for these

${ }^{1}$ Department of Medicine Division of Genetic Medicine, Vanderbilt University Medical Center/Vanderbilt-Ingram Cancer Center, Nashville, TN, USA; ${ }^{2}$ Department of Internal Medicine Division of Surgical Oncology, The Ohio State University, Columbus, OH, USA; ${ }^{3}$ Department of Clinical Genetics, Fox Chase Cancer Center, Philadelphia, PA, USA; ${ }^{4}$ Departments of Neurology, Neurobiology, and Cell Biology, Duke Center for Neurodegeneration \& Neurotherapeutics, Duke University Medical Center, Durham, NC, USA; ${ }^{5}$ Department of Breast Medical Oncology, The University of Texas MD Anderson Cancer Center, Houston, TX, USA; ${ }^{6}$ Departments of Obstetrics and Gynecology and Clinical Genomics, Mayo Clinic, Rochester, MN, USA; ${ }^{7}$ Department of Obstetrics \& Gynecology and Women's Health, Montefiore Medical Center, Albert Einstein College of Medicine, New York, NY, USA; ${ }^{8}$ Invitae Laboratory, San Francisco, CA, USA; ${ }^{9}$ Departments of Medicine (Medical Genetics) and Genome Sciences, University of Washington Medical Center, Seattle, WA, USA. Correspondence: ACMG (documents@acmg.net)

The Board of Directors of the American College of Medical Genetics and Genomics approved this statement on 15 October 2019. 
patients to access testing. Given the increasing and expanding importance related to testing breast cancer patients for inherited disease, the purpose of this points to consider document is to provide a rationale for maintaining support for existing evidence-based guidelines based on a risk stratification approach while data addressing broader testing strategies emerge.

\section{Practice guidelines and costs}

Practice guidelines issued through the National Comprehensive Cancer Network (NCCN) were originally developed over 20 years ago and are updated at least annually to incorporate new evidence to support revisions. ${ }^{13}$ These criteria stratify risk of hereditary breast and ovarian cancer syndromes based on personal and/or family cancer history to identify those at highest risk in whom to recommend genetic testing. NCCN includes recommendations that support risk-reducing mastectomy (RRM) as an option for patients who carry highly penetrant P/LP variants in BRCA1/2, PALB2, TP53, or PTEN.

When the original criteria were developed, testing was accomplished through Sanger sequencing, the BRCA1/2 genes were patented, and all clinical testing in the United States was conducted through a single laboratory at a cost of over $\$ 4000$ for testing of these two genes. Since that time, the switch to massively parallel sequencing (MPS) and the rescindment of the $B R C A 1 / 2$ gene patent in 2013 have led to industry competition with self-pay costs as low as $\$ 250$ per test or less. ${ }^{14}$ MPS testing platforms allow testing BRCA1/2 genes as part of multigene panels that include many other inherited cancer genes. Costs for multigene panels are often the same as single-gene testing. NCCN guidelines discuss the availability of multigene panel testing. Judicious use of laboratory test selection, discussion of variant penetrance and increased possibility of VUS, and pre- and post-test counseling with a trained genetics professional or health-care provider with special expertise in cancer genetics principles are all suggested.

\section{Genetic testing for all breast cancer patients?}

A recent study of 959 breast cancer patients who underwent an 80-gene panel test reported similar prevalence of P/LP variants identified in those patients who met NCCN guidelines for genetic testing compared with those who did not. ${ }^{15}$ However, the data upon which the conclusions of this study were based were limited by selection bias due to exclusion of those previously tested, uncertain accuracy of the recording of risk criteria for study participants, inclusion of genes with undefined actionability, and difference in patterns of genes in which P/LP variants were identified across the two groups. Further analysis of the data presented revealed that the prevalence of those meeting guidelines increased with penetrance of the gene in which the P/LP variant was identified. Among those patients who were heterozygous for $B R C A 1 / 2, T P 53$, and PTEN, 3\% met NCCN criteria compared with only $0.6 \%$ for those who did not meet criteria. When PALB2 was included, these proportions increased to 4.4 and
$0.8 \%$, respectively. The addition of moderately penetrant P/LP variants (e.g., ATM and CHEK2) led to further increase in frequency among those who did versus did not meet NCCN criteria ( $6 \%$ versus $3.3 \%$, respectively). Although diagnostic yield increased as more genes were tested, this increase disproportionately led to detection of P/LP variants in moderate penetrance genes, or genes in which association with breast cancer risk is uncertain. Soon after the publication of this paper, the American Society of Breast Surgeons published a recommendation to make genetic testing available to all breast cancer patients. This recommendation included genetic testing for "BRCA1/BRCA2 and PALB2, with other genes as appropriate for the clinical scenario and family history." 16

\section{DISCUSSION}

A critical analysis of the use of genetic testing to guide clinical care requires input from patients, health-care providers, laboratory geneticists, health-care systems, and public and private insurers. This discussion should also include gene penetrance, the constitution of multigene panels, and the clinical management associated with P/LP variants for genes included on multigene panels (e.g., RRM). Testing models should apply to patients already diagnosed and treated and those who may be at risk for disease.

\section{Multigene panels and variant penetrance}

The actual penetrance of P/LP variants in many (non-BRCA1/ 2) genes included on multigene panels is incompletely characterized. ${ }^{17}$ Currently breast cancer penetrance data are used to guide follow-up care after positive results (i.e., identification of P/LP variant). Follow-up may include either enhanced screening or RRM for genes with the highest penetrance. In contrast, only heightened screening (but not RRM) is recommended for other moderate penetrance genes that reach the threshold for it, based on a $\geq 20 \%$ lifetime risk for breast cancer. Clinical decision-making can be impacted depending on specific variants, especially when considering prophylactic surgical interventions. RRM is a consideration for those women with a P/LP variant in BRCA1/2, PALB2, TP53, and PTEN, recognizing that RRM recommendations for the latter 3 genes are inferred based on data among BRCA1/2 pathogenic variant carriers. ${ }^{18}$

There are robust data to support elevated cancer risk in the range of $25-30 \%$ associated with P/LP variants in ATM and CHEK2. ${ }^{19}$ This degree of risk meets the threshold for screening through breast magnetic resonance imaging (MRI); however, there are no data to support RRM based solely on P/LP variants that confer this level of risk. Furthermore, data suggest that the range of predicted risks for those who carry P/LP variants in ATM and CHEK2 likely includes a significant number of individuals who would not meet risk criteria for incremental screening. ${ }^{20}$ Additionally, $\mathrm{P} / \mathrm{LP}$ variants in moderate compared with high penetrance genes are more likely to be impacted by risk modifiers such as family history, single-nucleotide polymorphisms (SNPs), and 
mammographic density, as demonstrated by modeling studies. $^{21,22}$

There remain many genes on existing multigene cancer panels for which breast cancer risks are yet to be established or confirmed (e.g., RAD50, BRIP1, XRCC2, and MRE11A, among others). Furthermore, the data to support that NBN P/ LP variants increase breast cancer risk is limited to one variant (Slavic truncating founder variant, 657del5), ${ }^{19}$ with more recent studies indicating no association. ${ }^{23}$ It is also important to consider that as more genes are tested with sequencing methods, the complexity of results correspondingly increases. This is in part due to increases in detection of $\mathrm{P} / \mathrm{LP}$ variants identified in genes with uncertain cancer risks and lack of evidence to inform management, as well as higher chance of detecting variants of uncertain significance (VUS). ${ }^{24,25}$ Shared decision-making with patients and providers in the context of family history is recommended when discussing clinical management and risk-reducing surgery for $\mathrm{P} / \mathrm{LP}$ variants in moderate penetrance genes.

\section{Clinical management and precision medicine}

As progress in precision medicine continues, it is important that patients receive accurate information to ensure the implementation of risk-reducing strategies and evidencebased cancer genomics best practices. As noted above, clinical decision-making can be impacted depending on P/LP variants in specific genes. High rates of bilateral mastectomy were reported among breast cancer patients with a VUS after sequencing $B R C A 1 / 2 .^{26,27}$ These underscore the importance of genetics education for clinicians who perform genetic testing. There is potential for harm when genetic testing results lead to inappropriate management. In addition, reanalysis of VUS can be complicated because the process includes the ordering physician, the laboratory, and the patient. ${ }^{28}$ Furthermore, P/ LP variants associated with breast cancer may also be associated with other cancers, the interpretation and management of which needs to be addressed by the provider who orders the test. Health benefits of those tested should be maximized through early detection or prevention of cancer and treatment, while harms are minimized to ensure that patients receive guideline-adherent care.

\section{Evidence-based screening for cancer patients}

Recognizing the logistical challenges of identifying high-risk individuals most of whom are neither identified or tested, the recent US Preventive Services Task Force (USPSTF) guidelines for $B R C A 1 / 2$ testing highlighted specific evidence-based screening models to identify individuals suitable for referral and testing. ${ }^{29}$ Although simplifying guidelines is desirable, it remains important to identify appropriate candidates for testing based on the current evidence. While complexity of the NCCN criteria may represent a structural barrier to identifying breast cancer patients at risk for inherited disease, other policy-level factors such as mandates requiring genetic counseling prior to testing may present additional obstacles that disproportionately impact underserved and minority populations. $^{30}$ Consequently, simplifying criteria, reducing barriers to care, and expanding testing indications as supporting evidence evolves are appropriate. In fact, the most recently released NCCN guidelines focused on simplifying the criteria, and incorporated metastatic HER2-negative breast cancer as a testing criteria, given the emerging therapeutic implications based on identification of germline BRCA P/LP variants $^{3,4}$ which resulted in FDA drug approvals. As per national guidelines, in some subgroups (e.g., Ashkenazi Jewish heritage), all women with breast cancer are recommended to undergo genetic testing. ${ }^{13,29}$ Of note, a recent paper considered the cost-benefit ratio of multigene panel testing for all breast cancer patients ${ }^{31}$ and while the modeling results might be overly optimistic, these types of efforts are crucial in generating data to inform testing practices.

\section{General population screening?}

A broad discussion about population-based screening of unaffected women for a subset of inherited breast cancer genes is needed prior to considering genetic testing for all. ${ }^{32}$ There are similarities with the issues described but a more detailed discussion is outside the scope of this document. The population-based screening framework put forth by Wilson and Jungner in 1968 has some validity in this context although it was not intended to be used at the case level or applied to patients with an established condition and did not incorporate the many variables that are used to establish population risk. ${ }^{33}$ Furthermore, there are knowledge gaps when implementing multigene testing panels in this setting. These include penetrance of P/LP variants in specific genes and the natural history of P/LP variants in the genes being tested. Closing these gaps is fundamental to adopting population-based screening programs that adopt large panels.

\section{POINTS TO CONSIDER}

The American College of Medical Genetics and Genomics considers germline genetic information to be critical to the management of patients with genetic conditions, and advocates for the removal of barriers to patients gaining access to genetic information per clinical indications. We present points for clinicians to consider in the context of testing breast cancer patients for inherited cancer predisposition:

1. All patients with breast cancer should be evaluated to determine the need for germline genetic testing for hereditary breast cancer. According to national practice guidelines, genetic testing for breast cancer patients is indicated based on patient characteristics, including age at diagnosis, family cancer history, expression of estrogen progesterone receptors and HER2 expression, and disease stage. $^{13}$

2. When discussing genetic testing for breast cancer patients, clinicians should be aware of the current insufficient evidence to support genetic testing for all patients with breast cancer, especially with multigene 
panels that include genes without evidence to support follow-up care.

3. When discussing management after identification of a P/LP in moderately penetrant breast cancer genes:

a. Recognize that guidance is based on consensus recommendations.

b. Enhanced screening has not, to date, been associated with enhanced survival or identification of disease at an earlier stage.

4. Whenever genetic testing for inherited breast cancer is performed on a clinical basis, testing should include full gene sequencing, deletion/duplication analysis, and detection of known P/LP intronic variants in a CLIA-certified and/or College of American Pathologists (CAP)-accredited genetic testing laboratory.

5. The implications of genetic testing should be reviewed with patients in the context of genetic counseling as genetic testing is ordered. This counseling should include the expertise of a trained genetics professional or healthcare provider with special expertise in cancer genetics principles.

6. Patients who have a P/LP variant in an established breast cancer associated gene in which evidence-based follow-up recommendations exist should be educated about the importance of cascade testing of family members.

\section{Future considerations}

With the advances in sequencing technologies and increasing access to and expanding indications for genetic testing, it remains critical to ensure that implementation of testing is based on evidence. Currently, there is insufficient evidence to recommend genetic testing for $B R C A 1 / 2$ alone or in combination with multigene panels for all breast cancer patients. We expect that the evidence to support testing may evolve at different rates for different genes, and we expect that therapeutic indications will play a major role in the incorporation of genes to multigene panels. Consequently, as guidelines for testing are developed, it is critical to ensure they are supported by evidence and resources supporting strategies that include screening, medical, and/or surgical care as indicated. Ideally, professional societies should work together to weigh data, formulate, and harmonize evidencebased recommendations, and seek to reduce barriers to care. Testing alone will not improve outcomes but rather implementation of appropriate care following testing is required. Additionally, data are needed to generate evidence that informs clinical practice. This includes cost-benefit analyses that consider data on uptake of individual cancer risk management strategies and benefit from cascade family variant testing (given families rather than individuals are the unit of care when considering genetic testing) and refinement of penetrance estimates for moderate penetrance and incompletely characterized genes. Moreover, recognizing existing disparities in genetic testing across populations, the implementation of precision medicine approaches across oncology must also consider a means by which the promise of genetic testing for inherited cancer predisposition may be realized by all populations, regardless of race, ethnicity, and ability to pay.

\section{DISCLOSURE}

J.L. has grant research support from Novartis, Medivation/Pfizer, Genentech, GSK, EMD-Serono, AstraZeneca, Medimmune; is on the Speakers Bureau for MedLearning, Physician's Education Resource, Prime Oncology, Medscape, Clinical Care Operations; has received honoraria from UpToDate; and has memberships on advisory committees or review panels, board memberships, etc. for AstraZeneca and Pfizer (both uncompensated). E.D.E. is an employee of Invitae and has stock and other ownership interests in Invitae. The other authors declare no conflicts of interest.

Publisher's note Springer Nature remains neutral with regard to jurisdictional claims in published maps and institutional affiliations.

\section{REFERENCES}

1. American Cancer Society. Cancer facts \& figures 2019. Atlanta, GA: 2019.

2. Kurian AW, Ward KC, Howlader N, et al. Genetic testing and results in a population-based cohort of breast cancer patients and ovarian cancer patients. J Clin Oncol. 2019;37:Jco1801854.

3. Robson M, Im SA, Senkus E, et al. Olaparib for metastatic breast cancer in patients with a germline BRCA mutation. N Engl J Med. 2017;377:523-533.

4. Litton JK, Rugo HS, Ettl J, et al. Talazoparib in patients with advanced breast cancer and a germline BRCA mutation. N Engl J Med. 2018;379:753-763.

5. Drohan B, Roche CA, Cusack JC Jr, et al. Hereditary breast and ovarian cancer and other hereditary syndromes: using technology to identify carriers. Ann Surg Oncol. 2012;19:1732-1737.

6. Childers $\mathrm{CP}$, Childers KK, Maggard-Gibbons M, et al. National estimates of genetic testing in women with a history of breast or ovarian cancer. $J$ Clin Oncol. 2017;35:3800-3806.

7. Jones T, Lockhart JS, Mendelsohn-Victor KE, et al. Use of cancer genetics services in African-American young breast cancer survivors. Am J Prev Med. 2016;51:427-436.

8. Kurian AW, Griffith KA, Hamilton AS, et al. Genetic testing and counseling among patients with newly diagnosed breast cancer. JAMA. 2017;317:531-534.

9. Jagsi R, Griffith KA, Kurian AW, et al. Concerns about cancer risk and experiences with genetic testing in a diverse population of patients with breast cancer. J Clin Oncol. 2015;33:1584-1591.

10. Cragun D, Weidner A, Lewis $C$, et al. Racial disparities in BRCA testing and cancer risk management across a population-based sample of young breast cancer survivors. Cancer. 2017;123:2497-2505.

11. Manickam K, Buchanan AH, Schwartz MLB, et al. Exome sequencingbased screening for BRCA1/2 expected pathogenic variants among adult biobank participants. JAMA Netw Open. 2018:1:e182140.

12. Hall MJ, Reid JE, Burbidge LA, et al. BRCA1 and BRCA2 mutations in women of different ethnicities undergoing testing for hereditary breastovarian cancer. Cancer. 2009;115:2222-2233.

13. Genetic/Familial High-risk Assessment: Breast, Ovarian, and Pancreatic V.1.2020. Available from URL: https://www.nccn.org/professionals/ physician_gls/pdf/genetics_screening.pdf. [accessed Dec 4, 2019].

14. Cragun D, Kinney AY, Pal T. Care delivery considerations for widespread and equitable implementation of inherited cancer predisposition testing Expert Rev Mol Diagn. 2017:17:57-70.

15. Beitsch PD, Whitworth PW, Hughes K, et al. Underdiagnosis of hereditary breast cancer: are genetic testing guidelines a tool or an obstacle? J Clin Oncol. 2019;37:453-460. 
16. American Society of Breast Surgeons. Consensus guideline on genetic testing for hereditary breast cancer. 2019. https://www.breastsurgeons. org/docs/statements/Consensus-Guideline-on-Genetic-Testing-forHereditary-Breast-Cancer.pdf.

17. Tung N, Domchek SM, Stadler Z, et al. Counselling framework for moderate-penetrance cancer-susceptibility mutations. Nat Rev Clin Oncol. 2016;13:581-588.

18. Antoniou AC, Casadei S, Heikkinen $T$, et al. Breast-cancer risk in families with mutations in PALB2. N Engl J Med. 2014;371:497-506.

19. Easton DF, Pharoah PD, Antoniou AC, et al. Gene-panel sequencing and the prediction of breast-cancer risk. N Engl J Med. 2015;372:2243-2257.

20. Lee $\mathrm{K}$, Seifert BA, Shimelis $\mathrm{H}$, et al. Clinical validity assessment of genes frequently tested on hereditary breast and ovarian cancer susceptibility sequencing panels. Genet Med. 2019;21:1497-1506.

21. West $A H$, Blazer KR, Stoll J, et al. Clinical interpretation of pathogenic ATM and CHEK2 variants on multigene panel tests: navigating moderate risk. Fam Cancer. 2018;17:495-505.

22. Lee A, Mavaddat N, Wilcox AN, et al. BOADICEA: a comprehensive breast cancer risk prediction model incorporating genetic and nongenetic risk factors. Genet Med. 2019;21:1708-1718.

23. Couch FJ, Shimelis $\mathrm{H}, \mathrm{Hu} \mathrm{C}$, et al. Associations between cancer predisposition testing panel genes and breast cancer. JAMA Oncol. 2017;3:1190-1196.

24. Kurian AW, Ward KC, Hamilton AS, et al. Uptake, results, and outcomes of germline multiple-gene sequencing after diagnosis of breast cancer. JAMA Oncol. 2018;4:1066-1072.

25. Strande NT, Riggs ER, Buchanan $A H$, et al. Evaluating the clinical validity of gene-disease associations: an evidence-based framework developed by the Clinical Genome Resource. Am J Hum Genet. 2017;100:895-906.

26. Kurian AW, Li Y, Hamilton AS, et al. Gaps in incorporating germline genetic testing into treatment decision-making for early-stage breast cancer. J Clin Oncol. 2017;35:2232-2239.

27. Murray ML, Cerrato F, Bennett RL, Jarvik GP. Follow-up of carriers of BRCA1 and BRCA2 variants of unknown significance: variant reclassification and surgical decisions. Genet Med. 2011; 13:998-1005.

28. David KL, Best RG, Brenman LM, et al. Patient re-contact after revision of genomic test results: points to consider-a statement of the American College of Medical Genetics and Genomics (ACMG). Genet Med. 2019;21:769-771.

29. Owens DK, Davidson KW, Krist AH, et al. Risk assessment, genetic counseling, and genetic testing for BRCA-related cancer: US Preventive Services Task Force recommendation statement. JAMA. 2019;322:652-665.

30. Whitworth $P$, Beitsch $P$, Arnell $C$, et al. Impact of payer constraints on access to genetic testing. J Oncol Pract. 2017;13:e47-e56.

31. Sun L, Brentnall A, Patel S, et al. A cost-effectiveness analysis of multigene testing for all patients with breast cancer. JAMA Oncol. 2019 Oct 3; https://doi.org/10.1001/jamaoncol.2019.3323 [Epub ahead of print].

32. D'Andrea $E$, Marzuillo $C$, De Vito $C$, et al. Which BRCA genetic testing programs are ready for implementation in health care? A systematic review of economic evaluations. Genet Med. 2016;18:1171-1180.

33. Wilson JMG, Jungner G. Principles and practice of screening for disease. https://apps.who.int/iris/handle/10665/37650. Accessed 10 May 2019 